\title{
Clinical outcomes of adjuvant radiation therapy and prognostic factors in early stage uterine cervical cancer
}

\author{
Hyun Ju Kim, MD', Woo Joong Rhee, MD1', Seo Hee Choi, MD', Eun Ji Nam, MD², \\ Sang Wun Kim, MD2, Sunghoon Kim, MD², Young Tae Kim, MD², \\ Gwi Eon Kim, MD1, Yong Bae Kim, MD \\ Departments of ${ }^{1}$ Radiation Oncology and ${ }^{2}$ Obstetrics and Gynecology, Yonsei Cancer Center, \\ Yonsei University College of Medicine, Seoul, Korea
}

Purpose: To evaluate the outcomes of adjuvant radiotherapy (RT) and to analyze prognostic factors of survival in the International Federation of Gynecology and Obstetrics (FIGO) IB-IIA uterine cervical cancer.

Materials and Methods: We retrospectively reviewed the medical records of 148 patients with FIGO IB-IIA uterine cervical cancer who underwent surgery followed by adjuvant RT at the Yonsei Cancer Center between June 1997 and December 2011. Adjuvant radiotherapy was delivered to the whole pelvis or an extended field with or without brachytherapy. Among all patients, 57 (38.5\%) received adjuvant chemotherapy either concurrently or sequentially. To analyze prognostic factors, we assessed clinicopathologic variables and metabolic parameters measured on preoperative 18F-fluorodeoxyglucose (FDG) positron emission tomography/computed tomography (PET/CT). To evaluate the predictive performance of metabolic parameters, receiver operating characteristic curve analysis was used. Overall survival (OS) and disease-free survival (DFS) were analyzed by the Kaplan-Meier method.

Results: The median follow-up period was 63.2 months (range, 2.7 to 206.8 months). Locoregional recurrence alone occurred in 6 patients, while distant metastasis was present in 16 patients, including 2 patients with simultaneous regional failure. The 5 -year and 10-year OSs were $87.0 \%$ and $85.4 \%$, respectively. The 5 -year and 10-year DFSs were $83.8 \%$ and $82.5 \%$, respectively. In multivariate analysis, pathologic type and tumor size were shown to be significant prognostic factors associated with both DFS and OS. In subset analysis of 40 patients who underwent preoperative PET/CT, total lesion glycolysis was shown to be the most significant prognostic factor among the clinicopathologic variables and metabolic parameters for DFS.

Conclusion: Our results demonstrated that adjuvant RT following hysterectomy effectively improves local control. From the subset analysis of preoperative PET/CT, we can consider that metabolic parameters may hold prognostic significance in early uterine cervical cancer patients. More effective systemic treatments might be needed to reduce distant metastasis in these patients.

Keywords: Adjuvant radiotherapy, Uterine cervical cancer, Hysterectomy, Adjuvant chemotherapy

\section{Introduction}

Early stage cervical cancer can be effectively treated with radical hysterectomy or definitive radiotherapy. According to previous reports, the 5-year survival rates of the International Federation of Gynecology and Obstetrics (FIGO) stage IB-IIA

\footnotetext{
Received 7 May 2015, Revised 8 June 2015, Accepted 16 June 2015.

Correspondence: Yong Bae Kim, MD, Department of Radiation Oncology, Yonsei Cancer Center, Yonsei University College of Medicine, 50-1 Yonsei-ro, Seodaemun-gu, Seoul 120-752, Korea. Tel: +82-2-2228-8095, Fax: +82-2-2227-7823, E-mail: ybkim3@yuhs.ac

(c) This is an Open Access article distributed under the terms of the Creative Commons Attribution Non-Commercial License (http://creativecommons.org/ licenses/by-nc/4.0/) which permits unrestricted non-commercial use, distribution, and reproduction in any medium, provided the original work is properly cited.
}

www.e-roj.org 
cervical cancer patients who undergo surgery range from 83\% to $91 \%$, while those of patients treated with radiotherapy (RT) alone range from 74\% to $91 \%$ [1,2]. Although patients with early cervical cancer achieve relatively good survival rates, about $20 \%$ of all early cervical cancer patients still experience treatment failures.

To reduce treatment failure, adjuvant treatments have been applied in consideration of clinical and pathologic risk factors for recurrence. Several studies have identified several risk factors associated with increased recurrence and mortality, including pelvic lymph node metastasis, parametrial invasion, and positive surgical margins as high risk factors $[3,4]$. Moreover, patients with large tumor diameter, lymphovascular space involvement, or deep stromal invasion are considered to be at intermediate risk of recurrence [5-7].

The Gynecologic Oncology Group phase III trial (GOG 92) evaluated the benefits and risk of adjuvant pelvic RT aimed at reducing recurrence in women with stage IB cervical cancer treated by radical hysterectomy and pelvic lymphadenectomy $[6,7]$. In the study, adjuvant RT significantly reduced the risk of recurrence and prolonged progression-free survival (PFS) in intermediate-risk patients with cervical cancer. In regards to high-risk patients, a phase III study (GOG 109/SWOG 8797) demonstrated that addition of cisplatin-based concurrent chemotherapy to postoperative RT significantly improves patient survival [8].

Nowadays, functional imaging modalities, such as $18 \mathrm{~F}$ fluorodeoxyglucose (FDG) positron emission tomography/ computed tomography (PET/CT), are commonly used as optional imaging studies for initial tumor staging, optimization of treatment, and detection of tumor recurrence in patients with uterine cervical cancer. FDG-PET/CT can also be performed to evaluate treatment responses, since FDG uptake is correlated with the viable tumor burden $[9,10]$. Moreover, some studies have suggested that metabolic responses on FDG-PET/CT are associated with good survival outcome in cervical cancer $[4,11]$

In this study, we investigated the outcomes of adjuvant RT and analyzed prognostic factors of survival and prognostic value of metabolic parameters in FIGO IB-IIA uterine cervical cancer patients.

\section{Materials and Methods}

\section{Patients}

Between 1997 and 2011, a total of 350 patients diagnosed with uterine cervix cancer underwent curative hysterectomy with or without pelvic lymph node dissection (PLND) and para-aortic node sampling (PALNS), followed by adjuvant radiotherapy, at Yonsei Cancer Center, Yonsei University College of Medicine (Seoul, Korea). Among the treated patients, those who were diagnosed with pathologic FIGO stage IB-IIA uterine cervical cancer were included, while patients who received neoadjuvant chemotherapy or RT or who had a history of other malignancy were excluded from the study. Finally, a total of 148 patients were included and their medical records were reviewed retrospectively.

\section{Pretreatment evaluation}

The routine procedure for staging included a physical examination, common laboratory tests, standard chest radiographs, intravenous pyelograms, barium enemas, cystoscopies, and sigmoidoscopies. FIGO staging was determined according to baseline study findings, gynecologic pelvic exam, and additional imaging studies, such as CT, $\mathrm{MRI}$, or PET/CT. Metabolic parameters, such as maximum standardized uptake value (SUVmax), mean standardized uptake value (SUVmean), metabolic tumor volume (MTV), and total lesion glycolysis (TLG) for the primary tumor, were measured on preoperative FDG-PET/CT. The SUV of the tumor was normalized to that of the background level in the middle of the ascending aorta to minimize the confounding effects of inter-scan variations in SUV measurement. MTV was delineated by auto-threshold at 1.5 times the background level in the aorta, followed by knowledge based manual editing. Maximum and mean SUVs normalized to the background level were computed. TLG was calculated as the product of the lesion SUVmean and MTV.

\section{Surgery}

Table 1 lists the treatment details. All patients underwent radical hysterectomies. Patients who underwent conization or type I hysterectomy were excluded. PLND was performed in 136 patients (91.9\%), and para-aortic lymph node sampling/ dissection (PALNS/D) was performed in 88 patients (59.5\%). Risk factors, such as resection margin, lymphovascular invasion $(\mathrm{LVI})$, stromal invasion depth, and the extent of pelvic or paraaortic lymph node (PALN) metastasis, were documented in surgical pathology reports.

\section{Radiotherapy}

Adjuvant radiotherapy was initiated within 3-4 weeks after surgery and delivered to the whole pelvis field. Extended field radiotherapy or brachytherapy was performed as necessary based on the decision of the radiation oncologist. Especially, 
brachytherapy was performed to reduce external beam radiotherapy (EBRT) dose to 41.4-45 Gy giving sufficient dose for vaginal vault with brachytherapy in order to reduce late complication of EBRT. Extended field radiotherapy was performed for patients with para-aortic lymph node metastasis or high level pelvic node metastasis. The superior border of the whole pelvis field was at the L4-L5 interspace, and thus, included all of the external iliac and hypogastric lymph nodes. The inferior border was either at the lower border of the ischial tuberosity or the lower border of the obturator foramen depending on the extension of the vagina. The lateral portal anterior margin was plated at the pubic symphysis, and the posterior margin was at the S2-S3 interspace. Whole-pelvis and extended-field irradiation were performed in 135 patients (91.2\%) and 13 patients (8.8\%), respectively. External beam irradiation up to a median of $50.4 \mathrm{~Gy}$ (range, 39.6 to $54.0 \mathrm{~Gy}$ ) was delivered five times per week with a dose of 1.8 Gy per fraction. In 40 patients (27\%) treated with combined external irradiation and brachytherapy, high-dose rate intracavitary irradiation was applied using a remote afterloading system

Table 1. Treatment profiles $(n=148)$

\begin{tabular}{lc}
\hline \multicolumn{1}{c}{ Characteristic } & Value \\
\hline BPLND & $12(8.1)$ \\
No & $136(91.9)$ \\
Yes & \\
PALNS/D & $60(40.5)$ \\
No & $88(59.5)$ \\
Yes & \\
Adjuvant treatment scheme & $91(61.5)$ \\
RT alone & $57(38.5)$ \\
CRT & \\
RT field & $95(64.2)$ \\
WP & $40(27.0)$ \\
WP + Brachytherapy & $13(8.8)$ \\
Extended field & \\
RT Dose (Gy) & $50.4(39.6-54.0)$ \\
EBRT & $12.0(9.0-30.0)$ \\
Brachytherapy & $50.4(41.4-80.4)$ \\
Total & \\
Chemotherapy regimen & $38(66.7)$ \\
Cisplatin-based & $16(28.0)$ \\
Paclitaxel/carboplatin & $3(5.3)$ \\
Other &
\end{tabular}

Values are presented as median (range) or number of patients (\%). BPLND, bilateral pelvic lymph node dissection; PALNS/D, paraaortic lymph node sampling/dissection; RT, radiotherapy; CRT, chemoradiation therapy; WP, whole pelvis; EBRT, external beam radiotherapy. with Ir-192 as the source up to a median of 12 Gy (range, 9 to $30 \mathrm{~Gy})$ at 3-5 Gy per fraction.

\section{Chemotherapy}

Adjuvant chemotherapy was performed in 57 patients (38.5\%) either concurrently or sequentially according to the preference of the gynecological oncologist. A number of chemotherapy regimens were used, most frequently with cisplatin-based regimens in 38 patients (66.7\%). Weekly cisplatin was used in 12 patients (8.1\%), while cisplatin was sometimes used in

Table 2. Patient characteristics $(n=148)$

\begin{tabular}{|c|c|}
\hline Characteristic & Value \\
\hline Age (yr) & $48(28-80)$ \\
\hline \multicolumn{2}{|l|}{ pFIGO stage } \\
\hline $\mathrm{IB} 1$ & $97(65.5)$ \\
\hline IB2 & $22(14.9)$ \\
\hline$\| A 1$ & $24(16.2)$ \\
\hline$\| A 2$ & $5(3.4)$ \\
\hline \multicolumn{2}{|l|}{ Pathologic type } \\
\hline SCC & $103(69.6)$ \\
\hline Non-SCC ${ }^{a)}$ & $45(30.4)$ \\
\hline \multicolumn{2}{|l|}{ Tumor size $(\mathrm{cm})$} \\
\hline$<5$ & $119(80.4)$ \\
\hline$\geq 5$ & $16(10.8)$ \\
\hline Unevaluated & $13(8.8)$ \\
\hline \multicolumn{2}{|l|}{ Resection margin } \\
\hline Negative & $133(89.9)$ \\
\hline Positive & $15(10.1)$ \\
\hline \multicolumn{2}{|l|}{ LVI } \\
\hline Negative & $51(34.5)$ \\
\hline Positive & $97(65.5)$ \\
\hline \multicolumn{2}{|c|}{ Stromal invasion depth } \\
\hline$<1 / 3$ & $18(12.2)$ \\
\hline $1 / 3-2 / 3$ & $54(36.5)$ \\
\hline$>2 / 3$ & $67(45.3)$ \\
\hline Unevaluated & $9(6.0)$ \\
\hline \multicolumn{2}{|l|}{ Pelvic LN metastasis } \\
\hline Negative & $65(43.9)$ \\
\hline Positive & $71(48.0)$ \\
\hline Unevaluated & $12(8.1)$ \\
\hline \multicolumn{2}{|l|}{ PALN metastasis } \\
\hline Negative & $83(56.1)$ \\
\hline Positive & $5(3.4)$ \\
\hline Unevaluated & $60(40.5)$ \\
\hline
\end{tabular}

Values are presented as median (range) or number of patients (\%). FIGO, International Federation of Gynecology and Obstetrics pFIGO, pathologic FIGO stage; SCC, squamous cell carcinoma; LVI, lymphovascular invasion; PALN, para-aortic lymph node.

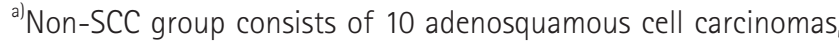
29 adenocarcinomas, and 6 mixed type. 
combination with vincristine (7.4\%), 5-fluorouracil (6.7\%), or other regimens (2.7\%). Paclitaxel/carboplatin combination therapy was used in 16 patients (28.0\%).

\section{Statistical analyses}

Overall survival (OS) was measured from the date of diagnosis to the date of death from any cause or the date at last clinical follow-up. Disease-free survival (DFS) was defined as the time between the date of diagnosis and the first recurrence of the disease or any death. OS and DFS were assessed using the Kaplan-Meier method. For multivariate analysis of prognostic factors, separate Cox proportional hazards regression models were utilized to estimate the relationship between each variables and OS or DFS. To evaluate the predictive

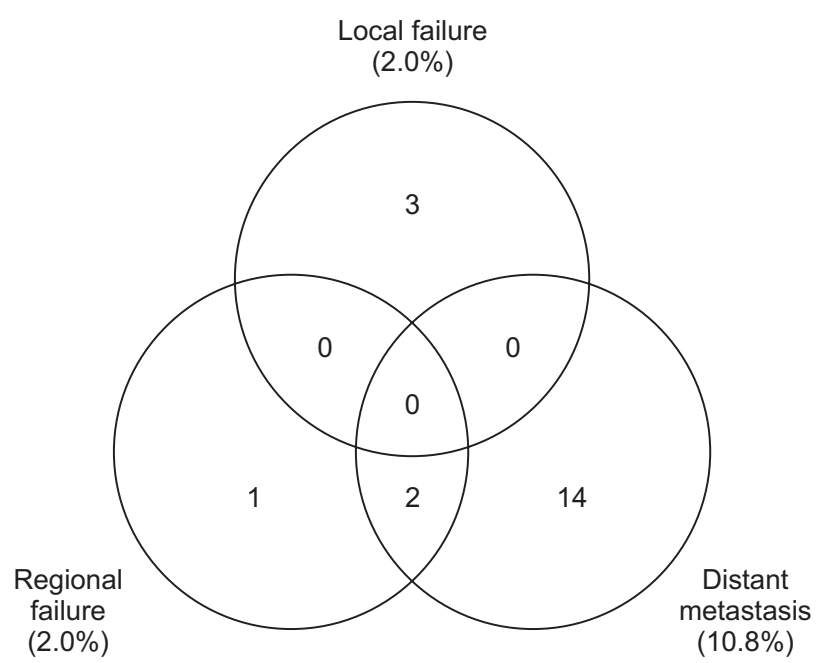

Fig. 1. Patterns of failures.

A

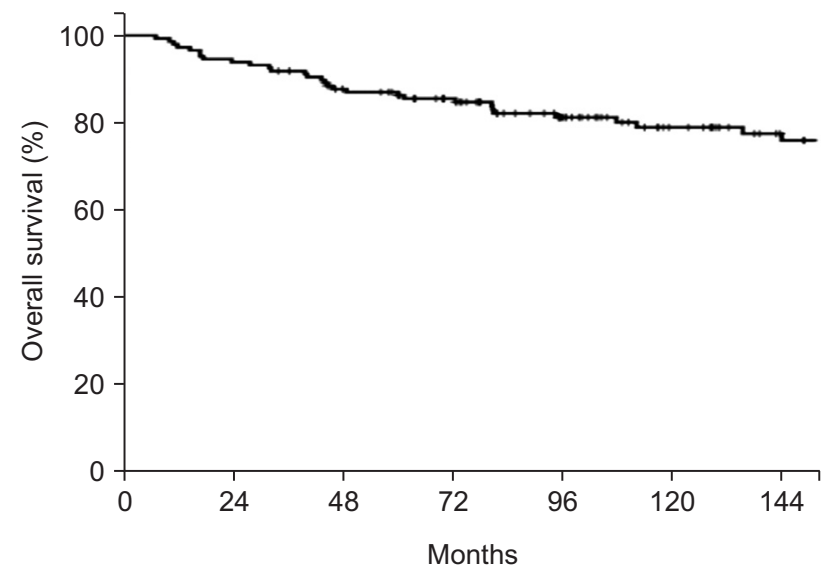

performance of metabolic parameters, we conducted timedependent receiver operating characteristic (ROC) curve analysis for censored survival data and assessed the area under the ROC curve (AUC). A two-sided p-value less than 0.05 was considered statistically significant.

\section{Results}

\section{Patient characteristics}

Patient characteristics are shown in Table 2. The median patient age was 48 years (range, 28 to 80 years). The majority of patients (97 of 148 patients, 65.5\%) were pathologic FIGO (pFIGO) stage IB1. Risk groups were defined using the same definitions used for the GOG phase III study (GOG 92) [6,7]. A total of 67 patients were categorized as the high-risk group and 81 patients were categorized as the low-intermediate risk group. The most common histopathologic type was squamous cell carcinoma (SCC, 69.6\%). Table 2 shows the details of patient characteristics including age, pFIGO stage, pathologic type, tumor size $(<5 \mathrm{~cm}$ or $\geq 5 \mathrm{~cm})$, resection margin, $L V I$, stromal invasion depth, and pelvic or PALN metastasis. Patients who did not undergo PLND or PALNS/D were assigned to the unevaluated group.

\section{Patterns of failures}

The median follow-up time was 63.2 months (range, 2.7 to 206.8 months). Twenty patients (13.5\%) experienced treatment failures after completion of their respective treatments. A detailed distribution of treatment failure patterns is shown in Fig. 1. Local failure occurred in 3 patients, while regional failure and distant metastasis occurred in 3 and 16 patients,

B

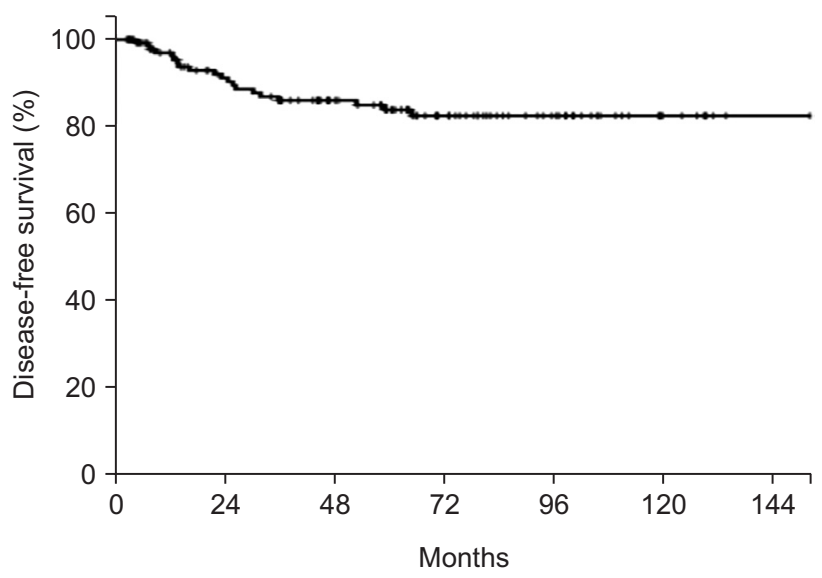

Fig. 2. Kaplan-Meier overall survival (A) and disease-free survival (B) curves. 
respectively. In 2 patients, both regional failure and distant metastasis were observed simultaneously.

\section{Survival outcomes and prognostic factors}

Among all of the patients, the 5-year and 10-year OSs were $87.0 \%$ and $85.4 \%$, respectively. The 5-year and 10-year DFSs were $83.8 \%$ and $82.5 \%$, respectively (Fig. 2). In univariate analysis, pathologic type, tumor size, and PALN involvement were significantly associated with DFS ( $p=0.075, p=0.005$, and $p=0.002$, respectively) (Table 3 ). OS was also affected by pathologic type, tumor size, and PALN involvement, the same as in the analysis of DFS. Different risk groups did not show statistically significant differences for either OS or
DFS. Similarly, the use of concurrent chemoradiation therapy (CRT) was not a statistically significant prognostic factor with respect to $0 \mathrm{~S}$ and DFS.

In multivariate analysis, pathologic type and tumor size were shown to be prognostic factors significantly associated with both DFS and OS. The hazard ratios (HRs) of non-SCC pathologic type and large tumor size $(\geq 4 \mathrm{~cm})$ were 3.78 (95\% confidence interval [Cl], 1.10 to $13.03 ; p=0.035)$ and $5.66(95 \%$ $\mathrm{Cl}, 1.56$ to $20.57 ; p=0.008)$, respectively.

\section{Prognostic value of metabolic parameters}

Preoperative PET/CT was performed in 40 of 148 patients. For metabolic parameters, such as SUVmax, SUVmean, MTV, and

Table 3. Prognostic analysis of disease-free survival and overall survival

\begin{tabular}{|c|c|c|c|c|c|c|c|}
\hline \multirow{3}{*}{ Variable } & \multirow{3}{*}{ Subgroup } & \multicolumn{3}{|c|}{ DFS } & \multicolumn{3}{|c|}{ OS } \\
\hline & & \multirow{2}{*}{$\frac{\text { Univariate }}{p \text {-value }}$} & \multicolumn{2}{|c|}{ Multivariate } & \multirow{2}{*}{$\frac{\text { Univariate }}{p \text {-value }}$} & \multicolumn{2}{|c|}{ Multivariate } \\
\hline & & & $\mathrm{HR}(95 \% \mathrm{Cl})$ & p-value & & $\operatorname{HR}(95 \% \mathrm{Cl})$ & p-value \\
\hline Pathologic type & SCC vs. non-SCC & 0.075 & $3.28(1.08-9.91)$ & 0.040 & 0.033 & $3.78(1.10-13.03)$ & 0.035 \\
\hline Tumor size $(\mathrm{cm})$ & $<4$ vs. $\geq 4$ & 0.005 & $3.23(1.03-10.09)$ & 0.044 & $<0.001$ & $5.66(1.56-20.57)$ & 0.008 \\
\hline Pelvic LN involvement & No vs. yes & 0.375 & - & - & 0.153 & - & - \\
\hline PALN involvement & No vs. yes & 0.002 & $2.51(0.50-12.67)$ & 0.265 & 0.007 & $1.78(0.33-9.73)$ & 0.506 \\
\hline Resection margin & No vs. yes & 0.593 & - & - & 0.934 & - & - \\
\hline LVI & No vs. yes & 0.312 & - & - & 0.064 & - & - \\
\hline CRT & No vs. yes & 0.857 & - & - & 0.627 & - & - \\
\hline Risk group & Low-intermediate vs. high & 0.837 & - & - & 0.394 & - & - \\
\hline
\end{tabular}

DFS, disease-free survival; OS, overall survival; HR, hazard ratio; $\mathrm{Cl}$, confidence interval; SCC, squamous cell carcinoma; LN, lymph node; PALN, para-aortic lymph node; LVI, Iymphovascular invasion; CRT, chemoradiation therapy.

Table 4. Prognostic factors for DFS in patients with PET/CT

\begin{tabular}{|c|c|c|c|c|}
\hline \multirow{3}{*}{ Variable } & \multirow{3}{*}{ Subgroup } & \multicolumn{3}{|c|}{ DFS } \\
\hline & & \multirow{2}{*}{$\begin{array}{c}\text { Univariate } \\
\text { p-value }\end{array}$} & \multicolumn{2}{|c|}{ Multivariate } \\
\hline & & & $\mathrm{HR}(95 \% \mathrm{Cl})$ & $p$-value \\
\hline Pathologic type & SCC vs. non-SCC & 0.009 & $6.56(0.67-63.75)$ & 0.105 \\
\hline Tumor size $(\mathrm{cm})$ & $<4$ vs. $\geq 4$ & 0.144 & - & - \\
\hline Pelvic LN involvement & No vs. yes & 0.741 & - & - \\
\hline PALN involvement & No vs. yes & $<0.001$ & $1.83(0.50-6.75)$ & 0.365 \\
\hline Resection margin & No vs. yes & 0.443 & - & - \\
\hline LVI & No vs. yes & 0.665 & - & - \\
\hline CRT & No vs. yes & 0.645 & - & - \\
\hline Risk group & Low-intermediate vs. high & 0.492 & - & - \\
\hline SUVmax & $<4.55$ vs. $\geq 4.55$ & 0.212 & - & - \\
\hline SUVmean & $<3.15$ vs. $\geq 3.15$ & 0.248 & - & - \\
\hline $\operatorname{TLG}\left(\mathrm{cm}^{3}\right)$ & $<191304$ vs. $\geq 191304$ & 0.001 & $18.745(1.30-270.24)$ & 0.031 \\
\hline $\operatorname{MTV}(g)$ & $<12.695$ vs. $\geq 12.695$ & 0.112 & - & - \\
\hline
\end{tabular}

DFS, disease-free survival; PET/CT, positron emission tomography/computed tomography; $\mathrm{HR}$, hazard ratio; $\mathrm{Cl}$, confidence interval; $\mathrm{LN}$, lymph node; PALN, para-aortic lymph node; LVI, Iymphovascular invasion; CRT, chemoradiation therapy; SUVmax, maximum standardized uptake value; SUVmean, mean standardized uptake value; TLG, total lesion glycolysis; MTV, metabolic tumor volume. 


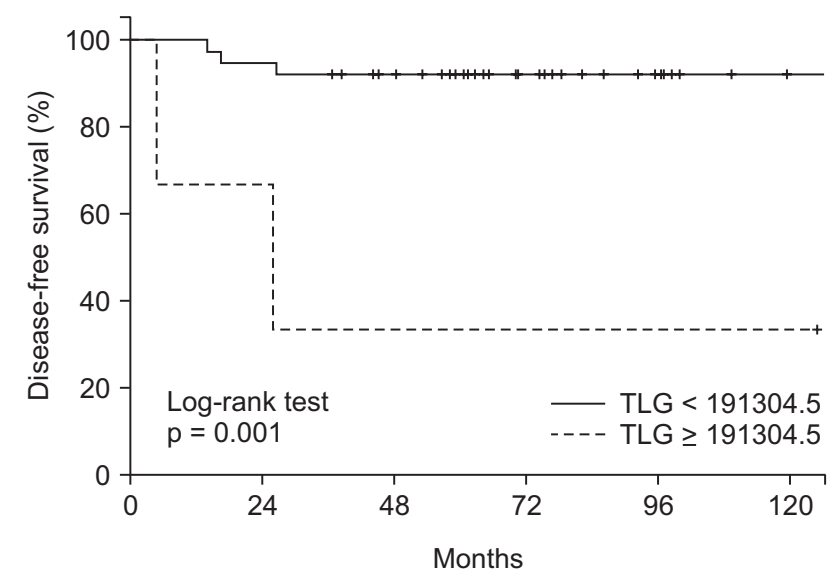

Fig. 3. Kaplan-Meier disease-free survival curve for total lesion glycolysis (TLG).

$T L G$, we intended to analyze their prognostic value. Among these patients, death occurred in only 1 patient, and recurrence occurred in 5 patients. Locoregional recurrence occurred in 2 patients, while distant metastasis was present in 4 patients, including 1 patient with simultaneous regional failure. The median SUVmax and the median SUVmean were 7.1 (range, 2.7 to 38.8) and 3.9 (range, 2.6 to 10.3). ROC analysis identified a cutoff of 4.55 for SUVmax (AUC $=0.583 ; p=0.135 ; 95 \% \mathrm{Cl}$, 0.318 to 0.848 ) and 3.15 for SUVmean ( $A U C=0.571 ; p=0.131$; $95 \% \mathrm{Cl}_{1} 0.314$ to 0.829 ). Cutoff values for MTV and TLG were measured as $12.695(\mathrm{AUC}=0.623 ; \mathrm{p}=0.137 ; 95 \% \mathrm{Cl}, 0.355$ to 0.891 ) and 191304.5 ( $A U C=0.629 ; p=0.151 ; 95 \% \mathrm{Cl}, 0.333$ to 0.925), respectively. Based on the ROC curve analysis, patients could be divided into two groups. In univariate analysis of clinicopathologic variables and metabolic parameters, pathologic type $(p=0.009)$, PALN involvement $(p<0.001)$, and TLG ( $p=0.001)$ were identified as prognostic factors for DFS (Table 4, Fig. 3). In multivariate analysis, TLG was the only prognostic factor for DFS ( $p=0.031 ; \mathrm{HR}=18.745 ; 95 \% \mathrm{Cl}, 1.30$ to 270.24). For OS, pathologic type $(p=0.001)$, tumor size $(p=$ $0.015)$, PALN involvement $(p<0.001)$, and TLG $(p=0.001)$ were identified as prognostic factors in univariate analysis; however, no variable was identified as significant in multivariate analysis.

\section{Discussion and Conclusion}

Radical hysterectomy with pelvic lymphadenectomy provides curative surgical excision and exhibits survival rates of more than $80 \%$ for stage IB and IIA cervical carcinoma. Adjuvant radiotherapy is thought to improve local control and overall survival in the remainder of patients. In our study, high locoregional control was achieved through adjuvant radiotherapy following surgery in early stage cervical cancer FIGO IB-IIA. Distant metastasis comprised the majority of treatment failures in our study. The addition of adjuvant chemotherapy to radiotherapy was not beneficial with respect to decreasing distant metastasis in our patients. These findings might be explained by the fact that the chemotherapy regimens were heterogeneous and the aim of concurrent chemotherapy with radiotherapy was not to improve systemic control but to achieve a radiosensitizing effect. Additionally, the number of patients who experienced distant failures was too small to show any statistically significant difference in our study.

In our study, pathologic type and tumor size were shown to be significant prognostic factors associated with both DFS and OS in multivariate analysis. This result is well correlated with several previous studies that reported on independent risk factors frequently associated with increased cancer recurrence and mortality [12-14]. In the present study, large tumor size was the most significant prognostic factor for worse OS, with a HR of 5.7, which is consistent with the results of other studies. Chung et al. [13] reported that cervical cancer larger than $4 \mathrm{~cm}$ in diameter determined by preoperative palpation was associated with a 5-fold increase in pelvic lymph node metastasis, a 10-fold increase in recurrence, and a 50\% decrease in survival. Additionally, in our study, non-SCC type was associated with a 3.8-fold increase in cancer specific death, compared to SCC type. Several previous retrospective studies have reported that patients with adenocarcinoma have a poorer prognosis than patients with SCC $[12,14-16]$, whereas other studies have found no difference in survival between these two subtypes $[1,5,13,17]$. We previously reported that the 5-year OS and PFS of adenocarcinoma are much worse than those for SCC [18]. Mabuchi et al. [19] also reported that adenocarcinoma shows worse survival than SCC in early stage cervical cancer patients with intermediate- and high-risk factors, regardless of the type of adjuvant radiotherapy used after radical hysterectomy.

We also analyzed the outcomes of different risk groups (low-intermediate vs. high-risk) and the effect of adding chemotherapy to adjuvant radiotherapy. The 5 -year OSs of the low-intermediate risk group and high-risk group were 89.2\% and $85.1 \%$, respectively. The high-risk group exhibited worse survival outcomes than the low-intermediate risk group; however, risk stratification was not identified as a significant 
prognostic factor in either univariate or multivariate analysis. Likewise, addition of chemotherapy was not a significant prognostic factor in OS or PFS. In the GOG 109 trial, the addition of concurrent cisplatin-based chemotherapy to RT significantly improved PFS and OS for high-risk early-stage cervical cancer patients who underwent radical hysterectomy and pelvic lymphadenectomy [8]. Several other retrospective studies also concluded that adjuvant CRT is beneficial in intermediate risk groups $[17,20]$. However, in the present study, adjuvant CRT failed to show a benefit on PFS and OS. This result might be due to including more patients from the high-risk group in the adjuvant CRT group, compared with the RT-only group (61.9\% vs. 34.9\%). The currently ongoing GOG 263 trial aims to determine if adjuvant CRT can significantly improve recurrence free survival, compared to radiation therapy alone, in intermediate-risk FIGO stage I-IIA cervical cancer patients.

We also evaluated the prognostic significance of metabolic parameters on preoperative PET/CT. In multivariate analysis, TLG was identified as the only prognostic factor for DFS, among the clinicopathologic variables and metabolic parameters, and no significant factor was identified for OS. Several studies have also investigated associations between prognosis and metabolic parameters on preoperative FDG-PET/ CT data in primary cervical tumor mass, lymph nodes, and the uterine body [3,21]. These previous studies have described SUV as a predictor of poor prognosis. However, since SUV values only represent metabolic activity, tumor size and SUV cannot be considered together. In our study, TLG was identified as a significant prognostic factor, suggesting that both metabolic activity and volumetric parameters are important to predicting prognosis. This result indicates that metabolic parameters may hold prognostic significance in early uterine cervical cancer patients. However, further well-designed large scale studies are needed to definitively conclude the prognostic value of these metabolic parameters.

This study has limitations that stem from its retrospective nature. The use of chemotherapy, chemotherapy regimens, and radiation doses were not uniform, which may have affected tumor responses, although no statistically significant differences were found. Also, treatment related toxicities could not evaluated objectively due to lack of medical records about toxicities. Since PET/CT was performed as an optional imaging study for uterine cervical cancer patients, PET/CT was performed in only 40 of our study patients. In these patients, only one patient died and only five recurrences occurred.
Due to this small number of events, it was difficult to assess significant factors associated with OS. Nevertheless, this study has its strengths, in that a large number of patients were analyzed and a long-term follow-up was achieved for these patients. Additionally, our results suggested the usefulness of metabolic parameters in early uterine cervical cancer patients, even though only a small number of patients were analyzed.

In conclusion, our results demonstrated that adjuvant RT following hysterectomy effectively improves local control; meanwhile, CRT was not effective in preventing distant metastasis in patients with pathologic FIGO stage IB-IIA uterine cervical cancer. Through an analysis of preoperative $\mathrm{PET} / \mathrm{CT}$, we could consider that metabolic parameters may hold prognostic significance in early uterine cervical cancer patients. More effective systemic treatments might be needed to reduce distant metastasis in these patients.

\section{Conflict of Interest}

No potential conflict of interest relevant to this article was reported.

\section{References}

1. Landoni F, Maneo A, Colombo A, et al. Randomised study of radical surgery versus radiotherapy for stage Ib-lla cervical cancer. Lancet 1997;350:535-40.

2. Perez CA, Camel HM, Kao MS, Hederman MA. Randomized study of preoperative radiation and surgery or irradiation alone in the treatment of stage IB and IIA carcinoma of the uterine cervix: final report. Gynecol Oncol 1987;27:129-40.

3. Xue F, Lin LL, Dehdashti F, Miller TR, Siegel BA, Grigsby PW. F-18 fluorodeoxyglucose uptake in primary cervical cancer as an indicator of prognosis after radiation therapy. Gynecol Oncol 2006;101:147-51.

4. Schwarz JK, Siegel BA, Dehdashti F, Grigsby PW. Association of posttherapy positron emission tomography with tumor response and survival in cervical carcinoma. JAMA 2007;298: 2289-95.

5. Delgado G, Bundy B, Zaino R, Sevin BU, Creasman WT, Major F. Prospective surgical-pathological study of disease-free interval in patients with stage IB squamous cell carcinoma of the cervix: a Gynecologic Oncology Group study. Gynecol Oncol 1990;38:352-7.

6. Rotman M, Sedlis A, Piedmonte MR, et al. A phase III randomized trial of postoperative pelvic irradiation in Stage IB cervical carcinoma with poor prognostic features: follow-up of a gynecologic oncology group study. Int J Radiat Oncol Biol 
Phys 2006;65:169-76.

7. Sedlis A, Bundy BN, Rotman MZ, Lentz SS, Muderspach LI, Zaino RJ. A randomized trial of pelvic radiation therapy versus no further therapy in selected patients with stage IB carcinoma of the cervix after radical hysterectomy and pelvic Iymphadenectomy: a Gynecologic Oncology Group Study. Gynecol Oncol 1999;73:177-83.

8. Peters WA 3rd, Liu PY, Barrett RJ 2nd, et al. Concurrent chemotherapy and pelvic radiation therapy compared with pelvic radiation therapy alone as adjuvant therapy after radical surgery in high-risk early-stage cancer of the cervix. J Clin Oncol 2000;18:1606-13.

9. Kim S, Oh SW, Kim JS, Kim KH, Kim YK. Prediction of response by FDG PET early during concurrent chemoradiotherapy for locally advanced non-small cell lung cancer. Radiat Oncol J 2014;32:231-7.

10. Seol KH, Lee JE. PET/CT planning during chemoradiotherapy for esophageal cancer. Radiat Oncol J 2014;32:31-42.

11. Siva S, Herschtal A, Thomas JM, et al. Impact of post-therapy positron emission tomography on prognostic stratification and surveillance after chemoradiotherapy for cervical cancer. Cancer 2011;117:3981-8.

12. Boyce J, Fruchter RG, Nicastri AD, Ambiavagar PC, Reinis MS, Nelson JH Jr. Prognostic factors in stage I Carcinoma of the cervix. Gynecol Oncol 1981;12(2 Pt 1):154-65.

13. Chung $C K$, Nahhas WA, Stryker JA, Curry SL, Abt AB, Mortel R. Analysis of factors contributing to treatment failures in stages IB and IIA carcinoma of the cervix. Am J Obstet Gynecol 1980; 138:550-6.

14. van Nagell JR Jr, Donaldson ES, Wood EG, Parker JC Jr. The significance of vascular invasion and lymphocytic infiltration in invasive cervical cancer. Cancer 1978;41:228-34.

15. Waggoner SE. Cervical cancer. Lancet 2003;361:2217-25.

16. Park JY, Kim DY, Kim JH, Kim YM, Kim YT, Nam JH. Outcomes after radical hysterectomy according to tumor size divided by $2-\mathrm{cm}$ interval in patients with early cervical cancer. Ann Oncol 2011;22:59-67.

17. Huang YT, Wang CC, Tsai CS, et al. Clinical behaviors and outcomes for adenocarcinoma or adenosquamous carcinoma of cervix treated by radical hysterectomy and adjuvant radiotherapy or chemoradiotherapy. Int J Radiat Oncol Biol Phys 2012:84:420-7.

18. Kim YB, Kim GE, Pyo HR, et al. Differential cyclooxygenase-2 expression in squamous cell carcinoma and adenocarcinoma of the uterine cervix. Int J Radiat Oncol Biol Phys 2004;60:8229.

19. Mabuchi S, Okazawa M, Matsuo K, et al. Impact of histological subtype on survival of patients with surgically-treated stage IA2-IIB cervical cancer: adenocarcinoma versus squamous cell carcinoma. Gynecol Oncol 2012;127:114-20.

20. Okazawa M, Mabuchi $S$, Isohashi $F$, et al. Impact of the addition of concurrent chemotherapy to pelvic radiotherapy in surgically treated stage IB1-IIB cervical cancer patients with intermediate-risk or high-risk factors: a 13-year experience. Int J Gynecol Cancer 2013;23:567-75.

21. Kidd EA, Siegel BA, Dehdashti F, Grigsby PW. The standardized uptake value for F-18 fluorodeoxyglucose is a sensitive predictive biomarker for cervical cancer treatment response and survival. Cancer 2007;110:1738-44. 